\title{
Our Solar Siblings: A high school focused robotic telescope-based astronomy education project
}

\author{
Fitzgerald, M.T. ${ }^{1 *}$, McKinnon, D.H. ${ }^{1}$, Danaia, L. ${ }^{2}$, Cutts, R. ${ }^{1}{ }^{3}$, Salimpour, S. ${ }^{4}$, Sacchi, M ${ }^{5}$
}

\begin{abstract}
In this paper, a robotic telescope-centric high-school level astronomy education project, Our Solar Siblings (OSS) is outlined. The project, an LCO official education partner, was formed as an institution-independent non-profit collaboration of volunteers officially in 2014 , although the first version of the curriculum materials and approach was initially first designed in 2010 . We outline the five goals of the project and the three approaches (formal classroom, independent student research and providing support to similar endeavours) we implement to pursue these goals. The curriculum materials, a central part of the project, are outlined as are their connections to various curriculum. The independent research project aspect and recent activity is presented. The article concludes with a brief update on the OSS evaluation which drives the educational design and the project's future directions as of 2017.

\section{Keywords}

robotic telescopes; practical science; group working; distance teaching

${ }^{1,5}$ Edith Cowan Institute for Education Research, Edith Cowan University, Joondalup, WA, Australia

${ }^{2}$ School of Teacher Education, Charles Sturt University, Bathurst, NSW, Australia

${ }^{3}$ St. Paul's Grammar School, 52 Taylor Rd, Cranebrook NSW 2749 Australia

${ }^{4}$ Deakin University, Victoria, Australia

${ }^{5}$ Our Solar Siblings, Australia

*Corresponding author: mfitzasp@gmail.com
\end{abstract}

\section{Introduction}

Our Solar Siblings ${ }^{1}$, was officially formed in 2014 as an institution-independent collaboration between the first three authors and has expanded to be an entirely volunteer run, not-for-profit, collaborative project. The decision that the Our Solar Siblings entity be separate and independent from any public or private institution was based on lessons learned from wide-scale reviews of astronomy student research and robotic telescope projects (Fitzgerald et al. 2014, Gomez and Fitzgerald 2017). The reviews revealed that projects, which succeeded over the long term, were those that were not reliant on short-term grant funding or on the vagaries of

\footnotetext{
${ }^{1}$ http://www.oursolarsiblings.com
}

internal institutional funding and decision bodies but rather those that were relatively low budget, run by volunteers and which were self-funded.

Our Solar Siblings is primarily an astronomy education project focusing on both formal in-class and independent student research use of robotic telescopes for education. The project is an official Education Partner of Las Cumbres Observatory (Brown et al., 2013) and typically utilises the $0.4 \mathrm{~m}$ and the $1 \mathrm{~m}$ telescopes with occasional requested spectra from the $2.0 \mathrm{~m}$ telescopes.

Much of the background research and rationale for the Our Solar Siblings project is published in a variety of journal articles while the image processing pipeline is described in Fitzgerald 2018. In this article, we provide an overview of the 
project, outline the project goals, educational approach, and share some of the evaluation results. Finally, we share the project's future directions.

\section{Project Design}

The project was formed as a potential partial solution to the stagnating perceptions of science in Australia (Danaia et al., 2013), the gap between the ideal and the actual science classroom (Danaia et al., 2017) and, more politically prominent, Australia's stagnating performance in science on international tests such as PISA and TIMMS (Thomson et al. 2016a; Thomson et al. 2016b). The project design is fluid and evolving as it encounters new problems and barriers but its major goal statements are to:

1. Involve the nontrivial use of real astronomical data from a real research grade telescope;

2. Increase students' understanding and appreciation for the universe around them, what it looks like, what its history is and where they are in it as far as we can currently ascertain;

3. Increase students' appreciation for the true methodology and approach of science in contrast to the general, currently poor, students' perceptions of school science;

4. Increase the probability of students choosing science, other than as a potential personal interest, as a topic for higher level study or as a potential future career path or, at the very least, help them discover they are actually interested in science; and,

5. Enable students, or a smaller subset that so desire, to take their research to a natural scientific conclusion. Sometimes even to result in a scientific publication. (Fitzgerald et al., 2015a)

The project aims to achieve these five goals through the following three broad approaches:
1. Facilitate and develop every day in-class teachers to use robotic telescopes to teach astronomy towards the end of compulsory science schooling. (Year 10 in Australia)

In this scenario, we are most likely acting at the interface between the "Citizen Science" and the "Working with real data" part of the Rebull Funnel (Rebull 2018). Classroom teachers are usually capable, albeit challenged initially for the first few runs through the material, of leading the students through this process with the provided materials and support. The early parts of the classroom implementation deal with students collecting their own images (Citizen Science) and the latter parts deal with supernova photometry (Working with real data). It is this formal aspect of OSS that is the most scaleable.

The teacher helps facilitate this with guidance from the project personnel. In reality, there is little direct guidance needed as the curriculum materials are written in such a manner as to embed the necessary professional development for the teacher within the flow of the text (e.g. Townsend et al. 2017). It has to be made clear that we are aiming at getting any teacher, regardless of skill or interest levels, capable of doing authentic and engaging astronomy in their classroom, not just those keener ones that arise naturally from the school landscape. In acknowledging that aim, we need to somehow address or, at the very least acknowledge we cannot address, numerous barriers in the classroom (Fitzgerald et al., 2017) that stop teachers from doing so.

It also needs to be acknowledged that astronomy, for the broader majority of teachers, is likely rushed through in a very transmissive or trivial manner or left until the end of the year but still checked off the box. We are eventually aiming for those teachers to come on board and do astronomy. The keen physics and astronomy teachers and the gifted and talented teachers are already ripe for the conversion and join OSS willingly and swiftly. For most teachers who teach astronomy once a year at a particular time of semester, their development 
and confidence in teaching OSS may take 3-5 years of gradually adding pieces to their everyday practice piece by piece. It is very common to hear from a given teacher at only one month in any given year. It is certainly not the case that we can just "build the telescope and they will learn" (Slater et al., 2014), but rather a sustained long-term provision of support and development to teachers as they incorporate it into their practice (slowly).

2. Direct mentoring of high school students undertaking independent research projects within a variety of contexts, both inside and outside the formal school curriculum.

In this scenario, we are likely working at the "Contributing real data" area of the Rebull Funnel. While these projects are original research, the research question itself and the broad methodology is typically well-known and well-designed by the OSS team. The students undertaking independent research projects are sometimes identified through the formal program while other times found through serendipity or word of mouth.

This approach involves an equal partnership between project personnel and the student. The teacher rarely plays an overly active role in the process but is copied in on all communications. For each project, where possible, a research scientist in the general field is asked to comment on the work at various points. While the project personnel can lead through the methodology and process easily, an expert in the field can quickly pick out simple common problems and provide direction where progress is uncertain or slow as well as comments on the final results.

Philosophically it would be great if all students could inquire in an open guided fashion on (relatively) their own timescale. However, even these independent research projects come with them an explicit (submission to a science fair or as part of formal school curriculum) or implicit (the student will run out of time and will move on to university and real life and have little time to continue) deadline. Science in the real world does not tend to run to schedule and neither do independent research projects but keeping things limited to a predefined time frame and deadline is a good idea!

3. Active and open development of scaffolds, mentoring and support for other projects using robotic telescopes in the classroom all around the world.

The five goal statements for students above are not just goals for OSS students, but goals for all students around the globe. Where possible, OSS tries to support other projects attempting to undertake similar goals by mentoring project personnel to mentor teachers in a similar manner as well as provide access to the OSS Pipeline, Curriculum Materials, Software and Tools.

\section{Curriculum Materials}

The curriculum materials are a core part of Our Solar Siblings. They provide capacity for the teacher to provide an authentic astronomy course utilising robotic telescopes in the everyday compulsory classroom and science curriculum as well as providing a mechanism to identify keen students who may like to undertake an independent research project. The initial materials were developed by Fitzgerald and McKinnon independently for use in the "Space to Grow" project (Danaia et al., 2012). The principles of the Educational Design are outlined in a more detailed paper (Fitzgerald et al., 2015a) but the broad approach is described here.

\section{Addressing the Curriculum}

Since the previous educational design paper, the curriculum in Australia has changed from a state-based to a national curriculum (Australian Curriculum Assessment and Reporting Authority, 2017). While there are still state differences in interpretation and implementation, the core Year 10 content strand for Earth and Space Sciences in Australia is now:

The universe contains features including galaxies, stars and solar 
systems, and the Big Bang theory can be used to explain the origin of the universe (ACSSU188)

There are also broader curriculum statements in Year 10 that are also addressed by the OSS Curriculum. The "Nature and development of science" statements are easily addressed as the history of astronomy tells a great story of models and theories contested over time. It is also heavily linked to technological advances in terms of telescope and camera instrumentation. They are as follows:

- Scientific understanding, including models and theories, is contestable and is refined over time through a process of review by the scientific community (ACSHE191)

- Advances in scientific understanding often rely on technological advances and are often linked to scientific discoveries (ACSHE192)

The two "Use and influence of science" statements below are not quite as easily addressed in the context of astronomy. Part of the elaboration of the first statement involves "recognising that the study of the universe and the exploration of space involve teams of specialists from the different branches of science, engineering and technology". This can also be addressed within OSS but should also be matched with a variety of other elaborations in other content strands as can be explored on the Australian Curriculum website. In particular, the Square Kilometre Array is a gigantic international collaboration for a major observatory in Australia and South Africa and Australia has also just joined a major collaboration, the European Southern Observatory as a partner.

The second statement is probably best done in the context of the teaching and research funding of the theory of evolution (ACSSU185), particularly in the United States or the current climate change issues which can be dealt with inside the Earth Science Content Strand (ACSSU189). Both content strands are also in the Year 10 Curriculum.
Astronomy, while quite fundamental, does not paint nearly as clear or direct a picture for this item.

- People use scientific knowledge to evaluate whether they accept claims, explanations or predictions, and advances in science can affect people's lives, including generating new career opportunities (ACSHE194)

- Values and needs of contemporary society can influence the focus of scientific research (ACSHE230)

There are a number of "Science Inquiry Skills" for Year 10 in the Australian Curriculum that are also addressed simultaneously while learning the content and exploring the human side of science. While also broader in scope, the direct connections of these curriculum statements to a hands-on, inquiry-based, astronomy are fairly straightforward to make (aside from field work or ethical issues). Hence, the Year 10 Australian curriculum inquiry skills statements covered by OSS are presented here with no elaboration:

- Formulate questions or hypotheses that can be investigated scientifically (ACSIS198)

- Plan, select and use appropriate investigation types, including field work and laboratory experimentation, to collect reliable data; assess risk and address ethical issues associated with these methods (ACSIS199)

- Select and use appropriate equipment, including digital technologies, to collect and record data systematically and accurately (ACSIS200)

- Analyse patterns and trends in data, including describing relationships between variables and identifying inconsistencies (ACSIS203)

- Use knowledge of scientific concepts to draw conclusions that are consistent with evidence (ACSIS204) 
- Evaluate conclusions, including identifying sources of uncertainty and possible alternative explanations, and describe specific ways to improve the quality of the data (ACSIS205)

- Critically analyse the validity of information in primary and secondary sources, and evaluate the approaches used to solve problems (ACSIS206)

- Communicate scientific ideas and information for a particular purpose, including constructing evidence-based arguments and using appropriate scientific language, conventions and representations (ACSIS208)

\section{Sequencing of the Curriculum}

The basic sequence of "classes" for the Year 10 Australian Curriculum that Our Solar Siblings suggests to directly address the curriculum is the following:

1. Allocate teams or individuals to work on a longer "Science as a Human Endeavour" presentation to research an allocated person/group and event in science history for sociocultural context as well as how science works.

2. What are telescopes? Why does their size matter? Why are some images from telescopes blurry and others not?

3. What are some of the major "pretty" objects out there in the universe?

4. Where are these "pretty" objects in the night sky and why?

5. Using images collected from LCO, how do we construct a colour image? What is an image, how is it constructed and what is 'colour'?

6. How do the size, shape and colour of galaxies differ?
7. How do we measure the distance to a galaxy? Measuring the distance to M101 using simple photometry of a single supernova (sn2011fe).

8. How do we measure the expansion of the universe? Measuring the distance to multiple galaxies using supernovae to create a Hubble Plot and estimate the age of the universe.

9. Hold the presentation class about the various people/groups and major events followed by a reflection on science as a human endeavour.

OSS presents a hybrid learning progression that does two things: it hits the curriculum requirements and develops enough data familiarity to either step into an independent research project if they are interested or to find out that they aren't that interested in astronomy after all! (Both are positives!). They will have undertaken simple photometry on a variable star (a supernova), have manipulated fits files and filters (and have made colour images such as those in Figure 1!), have explored patterns and differences amongst various astronomical objects and made some calculations from observational data. Part of the assessment is reflecting on their written and drawn descriptions of the universe prior to the beginning of the course to what they have learned at the end.

Extra curriculum material exploring dark matter, dark energy, the local cosmography of the universe and the larger cosmic web is provided for scientific and conceptual completeness, even though the curriculum does not at all call for these elements. From a scientific and cosmological perspective, they do need to be there to provide the "full" picture of the components of the universe, it size, shape, structure and age.

The previous NSW curriculum, which the original form of OSS was based upon had a very significant focus on stars as the core topic for the content area. While this NSW curriculum has receded into the distance, much of the materials designed fits very strongly with the NGSS (Schleigh et al., 2015), the International Baccalaureate (Cutts 2018) and 


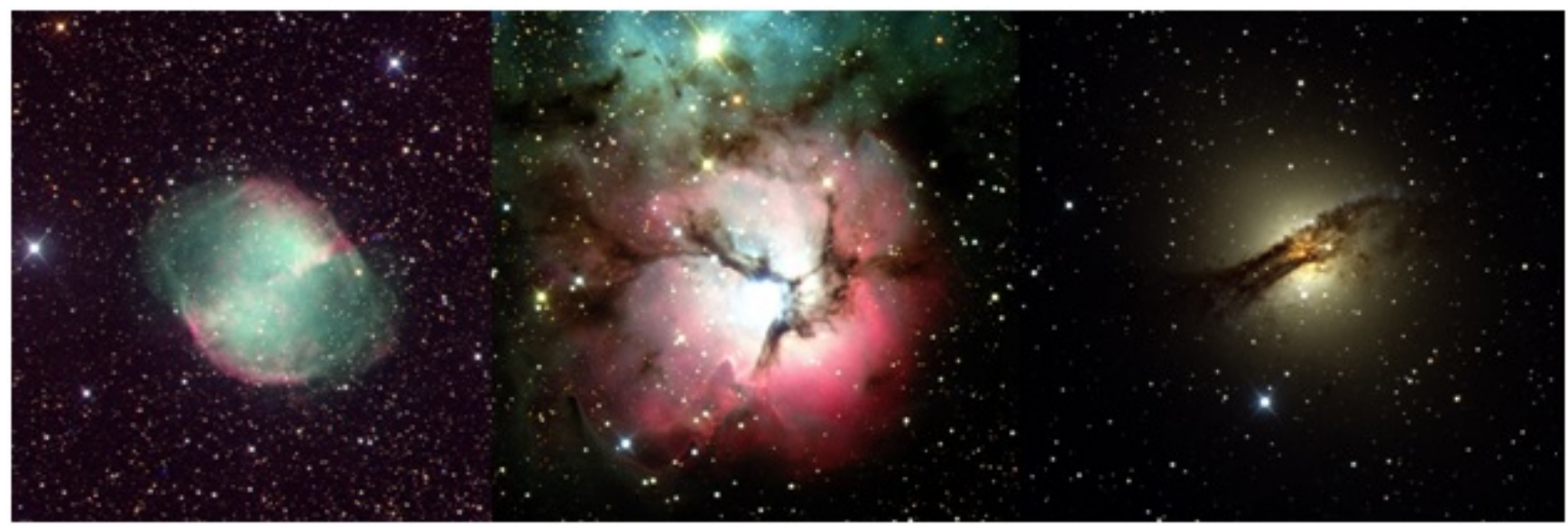

Figure 1. Sample colour images from three Our Solar Siblings students.

certain optional state-based extension and depth studies courses.

\section{Independent Research Projects}

The main scientific areas that Our Solar Siblings explores in science are related to stars or star-like point source objects. There are several reasons for this to be the case. One of the most basic reasons is that the measurement techniques for stars (effectively point sources) can be very reliably automated effectively from start to finish. As discussed in the OSS Pipeline paper (Fitzgerald 2018), there is only a finite amount of time in which to run these projects and there seems to be no great reason for students to undertake a silly-mistake-prone manual way of doing something that scientists routinely do automatically.

A very reasonable objection to this approach would be that an important part of the process is lost to the student. Perhaps so. This can be a decision made in cooperation with the student. The reality is though, time is short. If an Australian student is identified in Year 10 in normal science class and wants to do a project, then there really is only a single year in which to complete the project. This is because in Year 12, in just over a year, it is crunch time to score as high as possible to get the best university entrance score and next to no time will be allocated to the research project (unless it contributes to said score!).
If the student would like to spend the limited time learning about photometry manually and getting into the nitty gritty grind of manual image analysis for an eclipsing binary, then great! It is just a simple truth that after all these months of effort, the resulting light curve (likely unavoidably aperture photometry based rather than PSF photometry) will be of much poorer quality than that provided nearly instantaneously and silly-mistake-free by an automated pipeline. If the student has gone through OSS in class, they should already have undertaken photometry manually enough to have a good feel for what the numbers mean out of the other end.

The student will be interested in what they will be interested in, but all things being equal spending a year "Creating a UBVRI light curve set of an eclipsing binary" would be less exciting, less publishable and less scientifically useful than "Creating a physical model to estimate the mass and radii of the components of an eclipsing binary from UBVRI and spectroscopic data". It is unlikely that, with the students bumbling, flailing, experimental, error-prone steps while carrying a nearly boiling over cognitive load through such a guided-inquiry experience that *both* things can be done within a year. There is also the further question of attrition... the less you ask the student to do and the more exciting the topic, the less likely they will get overwhelmed and simply pull out of their project. But then again, maybe the student really is interested more in creating light curves than they are models. If it is a true guided inquiry 
model, then the student gets to heavily influence this choice of direction.

It is also the case that, more often as time goes on, high quality scientific-grade photometric data will become available from all-sky surveys in multiple epochs much of which will be far superior and reliable than what a student can achieve. This project is not a call for all student research to be using automated high quality precision photometry (or archival photometry, see Rebull 2018) but that this is an option. The raw images and data can always be made available if a student wants to go from absolute scratch in their project, but processed high quality data products from their requested images can also be available if that facilitates the success of the project. Where the student is placed in the reduction process is a discussion with the student but also the mentor's appraisal of the weight of the project load and the students' capacity to carry it.

\section{Some example projects}

Some of the most recent projects present good examples for matching the level of reduction to the scientific question and the students' capacity. One project (Cutts 2018) was to follow-up with observations of Hubbles' variable in M31, V1 (Templeton et al., 2011), the star that finally settled the Shapley-Curtis debate to show unequivocally that the "spiral nebulae" were truly outside our own galaxy. In this project, a student followed a single Cepheid over the course of months to plot it's light curve. As this was a single star, it was chosen that in-depth manual differential aperture photometry would be undertaken with Aperture Photometry Tool (Laher et al., 2012). Most of the work involved processing and checking in detail the data collected. The analysis of the light curve at the end being quite straight forward: Find the Period, Measure the apparent magnitude in BVI, simultaneously estimate the reddening and distance using the BVI data and the Cepheid Period-Luminosity Relationship. This is something entirely feasible within the nine month prescribed time period.
There are a number of projects (e.g. Cutts 2018) observing RR Lyraes and their behaviour in the SDSS filter system, where a theoretical pseudocolour-based period-luminosity-metallicity relationship shows some promise (Catelan et al. 2012, Cáceres and Catelan 2008). These projects include looking at the globular clusters, Messier 2, NGC1261 and Messier 15. Aside from the very diffuse globular cluster NGC6101 studied in Fitzgerald et al. (2012) by a pair of Year 11 students, studies of RR Lyrae stars in these clusters necessitates the use of PSF photometry inaccessible to students both in terms of available Operating System but also complexity of setup and procedure.

Even if manual aperture photometry was viable for some of these projects, the sheer number of images to be processed would be quite overwhelming and prohibitive in terms of time. Looking at RR Lyrae itself as a test took many short exposures in the 5 different filters with a total of 1076 short exposure images. Messier 2, with longer exposures on the 1-metre telescope had a total of 187 images but over 56 different variables per image with a similar number for ngc1261. A simple Jython \& Python script used by the students written by, a then student, automates the extraction of photometry for each star from thousands of images in minutes from the provided PSF photometry files. For the single M31-V1 star where aperture photometry was used carefully and with much focus, for the study of RR Lyrae with thousands of images, to the study of many multiple variables in a globular cluster, each project is a manageable and finite entity.

Precision photometry of Open Clusters is also a core research project within OSS. While there has only been one paper published about an open cluster in the project (Fitzgerald et al., 2015b), there are a variety of scientific papers on various Open Clusters waiting in the queue to be submitted. Open Clusters, as a topic, are a wonderful student research project because they are somewhat simple, yet each object is complex and unique and the topic captures much of the fundamentals of observational astronomy. They are also surprisingly little studied 
(Dias et al. 2002 (2016 catalogue)) with only about 400 having reliable CCD photometric data studies published. Even astronomers are surprised to know that there are only about 70 or so open clusters that are considered very well known (Paunzen and Netopil, 2006) but that most have not been studied to any great depth photometrically. There is a significant amount of interest in galactic archeology via the collection of huge numbers of abundant high resolution spectra, but the support of photometrically determined distance, reddening, age and metallicity has been relatively lacking.

In the provided Project 3 of the OSS materials, there is a lot of material that leads students slowly through the fundamentals of observational photometric astronomy, through which they learn about how to make a colour-magnitude diagram from scratch and interpret it in detail. They begin with pattern matching between provided Colour-Magnitude Diagrams (CMD) and their respective images to get a feel for the representation and then are dumped in the deep end making their own calibrated CMD. Depending on the level of the class or group, this can be a simple BV CMD of an open cluster with minimal reddening, usually m67 or NGC2420, or a full UBVRI treatment of an open cluster, NGC654, with significant reddening (roughly $\mathrm{E}(\mathrm{B}-\mathrm{V})=0.9$ ) using Colour-Colour diagrams as well to estimate the colour excess.

At this level, a large group of students are intended to collaborate in measuring the stars in the cluster via cutting up the cluster image into regions and typically pairs of students (a measurer and recorder) manually measure the stars using Makali'i, Aperture Photometry Tool (Laher et al., 2012) or AstroImageJ (Collins et al., 2017). This endeavour is heavily spreadsheet driven (see Figure 2) so that all of the calculations can be made apparent to the student, although the formulas and calculations are already embedded in the spreadsheet and the graph automatically plotted. Knowing the amount of small errors that the authors made with their relatively long experience, this could only be amplified dramatically if a student needed to create such a spreadsheet from scratch. In fact, creating the spreadsheet used here would be an entire project by itself! (Although if it were a true student research project, we would use python rather than Excel.)

The results from the spreadsheet are very robust and, with adequate calibration stars, return distance and reddening very close to the values derived in the professional literature. This is as true for the distance, where a simple ZAMS line is used to fit to the main sequence, as it is for the $(\mathrm{U}-\mathrm{B}) /(\mathrm{B}-\mathrm{V})$ Colour-Colour diagram where similar reddenings are observed. A screenshot of the final CMD from one classes' analysis of m67 is shown in Figure 3. It looks good! We can roughly estimate the age using the turn-off point and explore why stars are where they are on the diagram and go into great detail about stellar properties and evolution, which takes up a significant fraction of OSS Project 3. Working on their own data does provide a motivation to push through!

The photometry and resulting diagrams from this method would not be considered a scientifically publishable outcome though, although certainly much better than could ever have been achieved a decade or two ago. Things have moved forward. At the very least, in a class of 24-32 students, there will be innumerable copying and entering errors to begin with. Also, the calibration method using a single star with known UBVRI magnitudes to calibrate all the stars in the image is educationally quite useful and certainly good to plus or minus a few percent. It, however, certainly would not be the approach used to undertake precise calibrated photometry where an all-sky solution for each star in the image separately would be used. This is typically a nightmare to do manually, even for an expert, but something the OSS pipeline deals with quite well in an automated fashion.

Methodologically, there are issues with the manual aperture photometry undertaken as it is taken with a very non-optimal aperture. The optimal aperture for highest $\mathrm{S} / \mathrm{N}$ is $r \approx 0.68 \mathrm{FWHM}$ (Mighell, 1999), which only captures approximately $72 \%$ of the flux but the missing flux is extrapolated using 

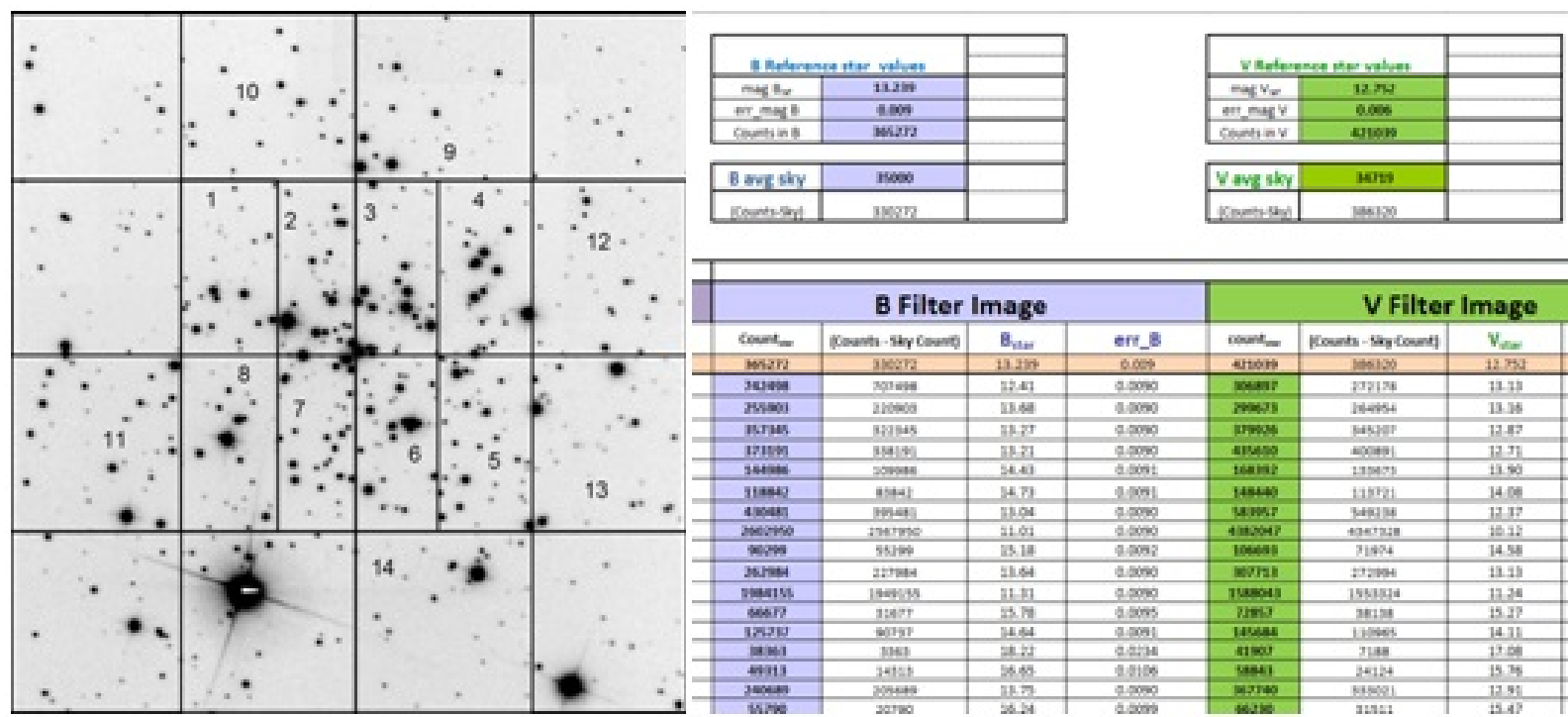

Figure 2. Finder chart for open cluster, NGC659 (left) and part of the spreadsheet used to create the colour-magnitude diagram (right)

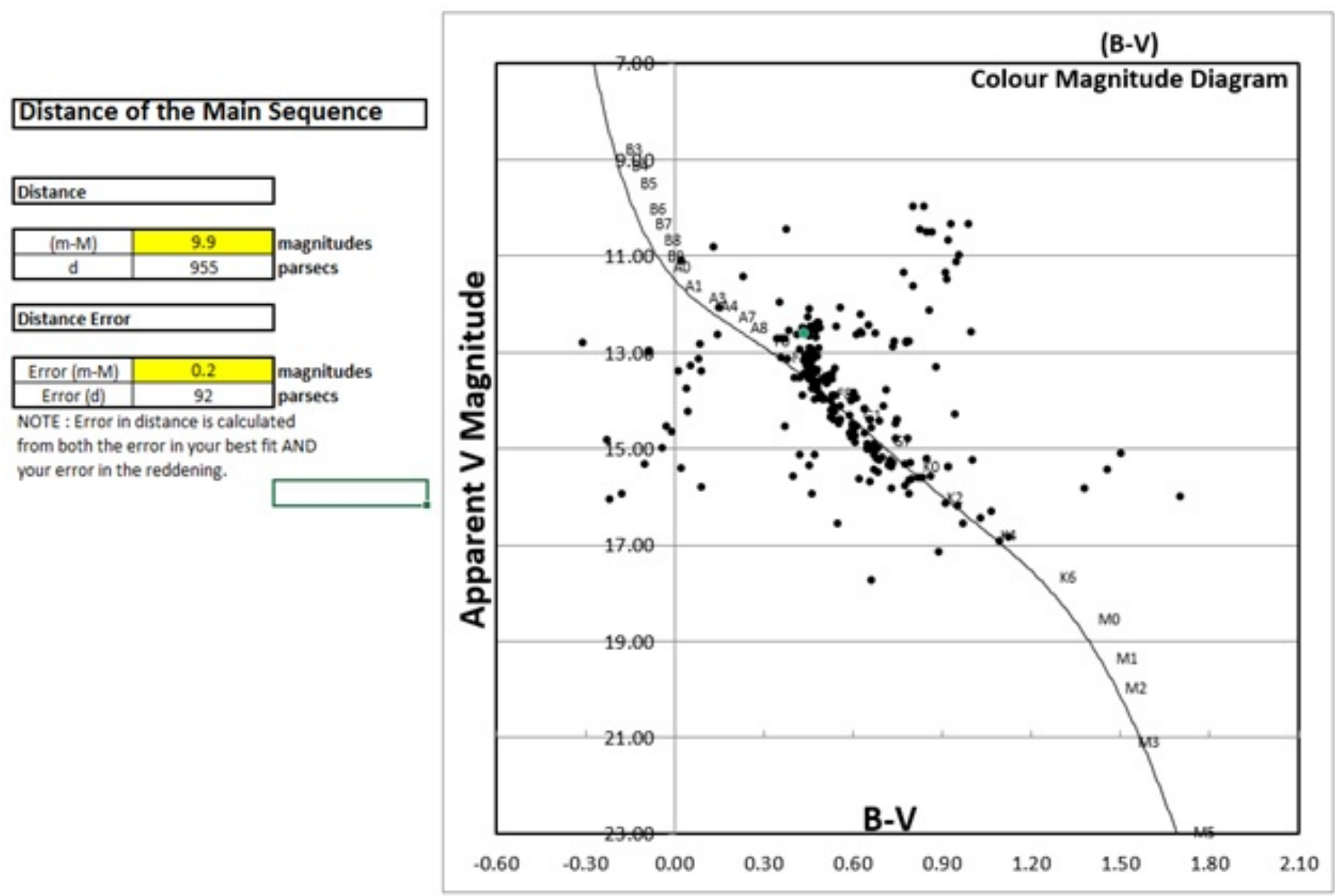

Figure 3. Final Colour Magnitude Diagram from a class for m67.

the curves of growth aperture corrections method. In contrast, in this application, students typically use an aperture radius multiple times the FWHM to capture most of the flux but, in doing so, also include a significant amount of noise from the wings of the star. Most of the time this is because 
the software package does not offer the option to do aperture correction measurements. In the case where it does (Aperture Photometry Tool), for this case it is not entirely necessary for this application where the goal is simply for students to learn the ropes.

For students who would like to undertake investigations with scientific grade photometry, the students use the calibrated all-sky output catalogue from the OSS Pipeline. This is usually either DAOPhot PSF based (Stetson, 1987) or Aperture Photometry Tool based and cross-matched to a variety of catalogues. The catalogue can then be opened in a piece of software called "Pysochrone" written by the first author. A screen shot of this software is shown in Figure 4.

Using the UBVRI (or ugriz) optical data from the students, matched with 2MASS and WISE infrared data and UCAC4 (or PPMXL or, soon, GAIA) proper motions, students can adjust the age, reddening and distance by changing values using the keyboard and fitting (Girardi et al. 2002, 2004) isochrones across multiple CMDs and Colour-Colour diagrams. The photometric metallicity can also be estimated by using the ultraviolet excess method initially (Karataş and Schuster, 2006) to get a ballpark value and the value finetuned along with the other four major parameters. Data cuts can also be made in terms of cluster size, membership probability from proper motions/radial velocities and photometric quality cuts.

Once the student has explored their fits to the data, using their finely-tuned pattern recognition tools (otherwise known as the eye), their estimates are checked against a subjectivity-free weighted likelihood fitting technique (Monteiro et al., 2010). The interaction between the objective fitting and what can be seen with the human eye can typically lead to a back and forth process between the student and the objective software as it illuminates potential problems in the data or potential biases within either approach. The final values and errors are taken from the objective fitting technique while acknowledging the visual fitting technique played a key part in quality control and interpretation.

Not all independent research projects involve stars. If a student wants to do a project but is unsure of their interests, they will be pointed towards one of the types of projects above. If the student already has an inkling of what they may be interested in, there are all manner of projects the students can undertake if their interests head in a particular direction. As long as they are largely optically based (if the student intends to observe using telescopes) and can plausibly be undertaken in a reasonable amount of time at a level where the student has some grasp and intuition about what is going on, then these topics are fair game.

The new custom topic will likely lack the scientific sophistication (and known direction towards publication) of the developed topics above and the road through the project may be bumpier, but the motivation and tenacity on the side of the student is increased. In this sense, it is closer to the idyllic open inquiry where the student has generated the actual question to be asked, the typical endpoint of Backward Faded Scaffolding (Slater et al., 2008), although much guidance is still necessary, perhaps even more so, on the actual methodology of the project.

A couple of examples of custom topics is the measurement of $\mathrm{H} 0$ using images of galaxies. This topic came out of the student's interest in the topic sparked by previous experience in Skynet Junior Scholars. Most of the work was undertaken by the student with periodic guidance from OSS. Another custom topic was looking for variable stars in NGC659 inspired by an article the student found related to an open cluster he explored in class. A previous jAAVSO paper (Souza, 2013) identified two new cool variables which were included in his study. The student wrote some Jython/STILTS/Python code to automate the star extraction from the photometric catalogues which is frequently used in research projects now.

\section{Evaluation}

A version of the materials and approaches used in Our Solar Siblings were employed in a previous 


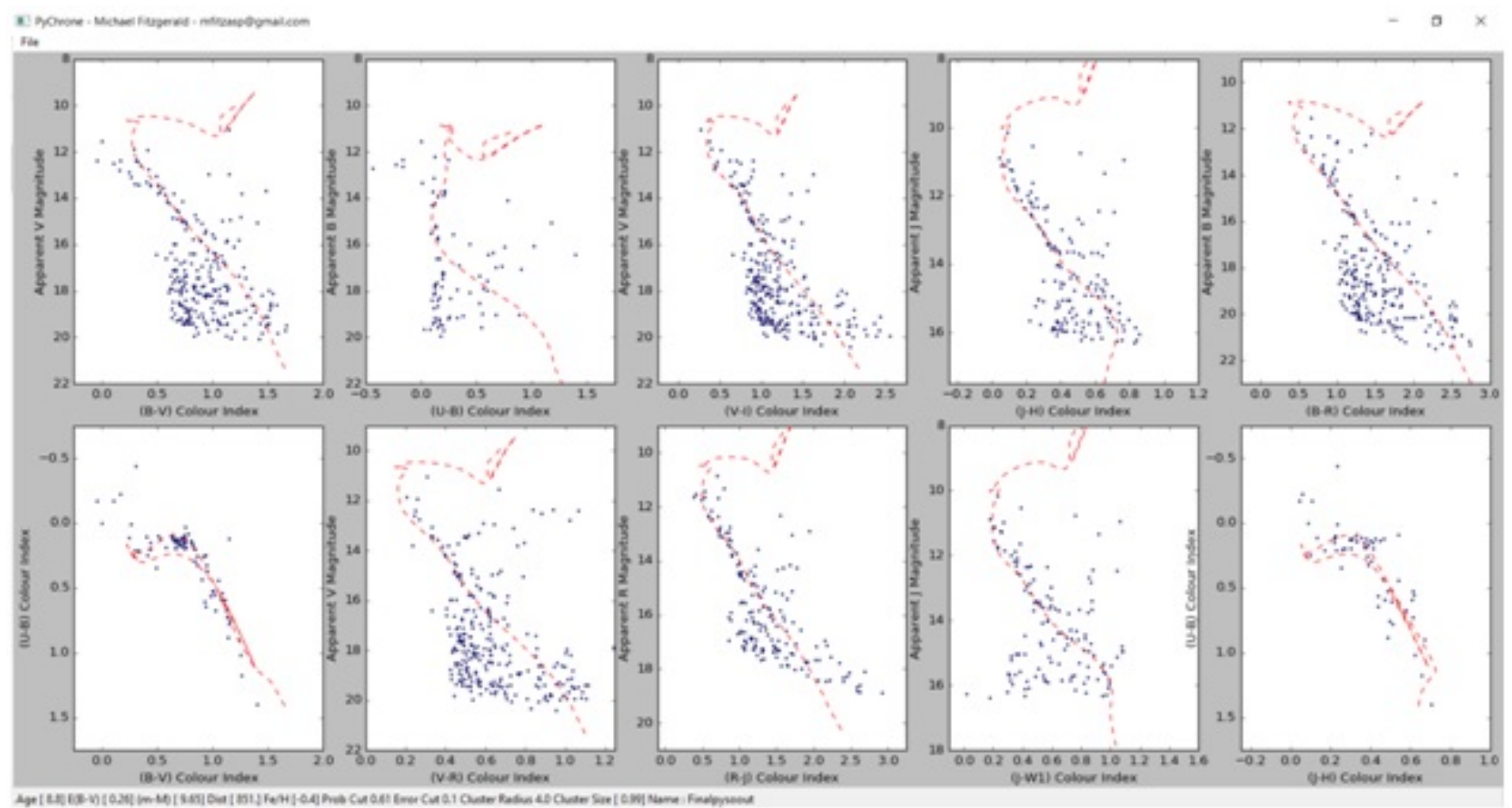

Figure 4. Screenshot of pysochrone software

project. OSS has significantly developed (and the curriculum entirely changed) since then! Our earlier evaluations (Fitzgerald et al., 2016) showed that we were doing quite well (statistically significant moderate strength impact factor) in teaching the actual content of the course to students as measured by a custom concept inventory. This has continued into the present era where we are getting even higher strength gains (an example shown in Figure 5) using an expanded version of the previous concept inventory (used in Lazendic-Galloway et al. 2017).

It seems, for the current moment, we are on the right path as far as basic conceptual content knowledge is concerned. While it is nice to have some success (and have data to prove it!), it could equally be said that simple content knowledge is both the easiest thing to shift and perhaps one of the less important aspects of the five goals of Our Solar Siblings.

The results from the earlier project in terms of student perceptions of their classroom as well as their perceptions and attitudes towards science were mixed with mostly minor, relatively random, positive and negative shifts in most aspects, although a few major positive shifts here and there (Fitzgerald et al., 2016). There was no solid conclusion as to why this was the case.

One aspect of the problem was undoubtedly the educational design, which at that point was very heavily content focused but not well developed to address students' more personal or cultural appreciations of science. As stated as an intention in the discussion of Fitzgerald et al. (2016), since then we have implemented many improvements into the educational design, in particular, incorporating more personal reflection and "science as a human endeavour" aspects into the design.

We have not yet evaluated the efficacy of these perception and attitude based modifications. This is because the other major aspect of the problem was that the attitude/perception survey that was used was a fairly blunt instrument and not well suited to what we were trying to measure. A new instrument has been developed to help measure these aspects (Bartlett et al., 2018) and evaluation of the new design will hopefully begin in the 2018 school year. This research will also look at potential shifts in 


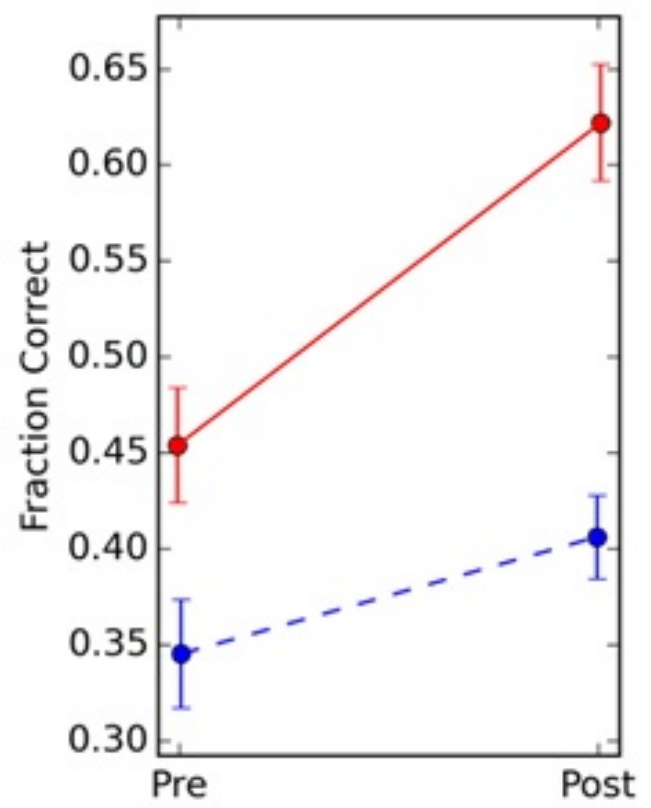

\begin{tabular}{|c|c|c|c|c|c|}
\hline $\begin{array}{l}\text { Descriptive } \\
\text { Statistics }\end{array}$ & Mean & St.Deviotion & $N$ & & \\
\hline $\begin{array}{l}\text { Equivalent Pre } \\
\text { Equivalent Post }\end{array}$ & $\begin{array}{l}45 \% \\
62 \%\end{array}$ & $\begin{array}{l}17 \% \\
17 \%\end{array}$ & $\begin{array}{l}31 \\
31\end{array}$ & $\begin{array}{l}31 \% \\
1.01 \\
\end{array}$ & $\begin{array}{l}\text { Percentoge Goin } \\
\text { Effect Size (Cohen's D) }\end{array}$ \\
\hline $\begin{array}{l}\text { Non-Equivalent Pre } \\
\text { Non-Equivalent Post }\end{array}$ & $\begin{array}{l}35 \% \\
41 \%\end{array}$ & $\begin{array}{l}16 \% \\
12 \%\end{array}$ & $\begin{array}{l}31 \\
31\end{array}$ & $\begin{array}{c}9 \% \\
0.44\end{array}$ & $\begin{array}{l}\text { Percentoge Goin } \\
\text { Effect Size (Cohen's D) }\end{array}$ \\
\hline
\end{tabular}

The Percentoge Gain for each Pre/Post pair is the Actual Gain (Post-Pre) divided by the largest possible gain achievoble (100\%-Pre). A goin of 100\% means that everybody in the class correctly answered all of the questions they got incorrect on the pre-test and got all of the pre-test questions correct again. This is obviously impossible? As this Gain is actually an internal property of the survey itself, we are still collecting information on typical values in different contexts so cannot provide a strong guidance as to what is a 'good" goin is as yet. However in similarly constructed survers,

Troditional" cholk'n'tolk classrooms tend to ochieve about a $10 \%$ gain whilst inquiry-based/lipped/etc. classrooms can range from $25 \%$ to $40 \%$ and beyond.

The Effect Size (Cohen's D) is a mare general estimate of how dramotic the change is. A broad introduction is avallable here: $h t t p: / / r p s y c h o l o g i s t . c o m / d 3 / c o h e n d /$ It compares the difference in the means from the pre te post to their standard deviations. Roughly speaking 0.2 is a small, 0.5 is a medium, 0.8 is a large and 1.3 is a huge effect size.

Figure 5. Current content knowledge gains for a recent OSS Year 10 class.

career intentions of students involved in the course.

\section{Achievement of Goals and Future Directions}

In this section, we briefly comment on whether the stated goals are being achieved and outline the future directions for the project.

- Involve the nontrivial use of real astronomical data from a real research grade telescope;

This certainly has been achieved, largely through the use of the OSS Pipeline to automatically process and deliver high quality data products to the teachers and their students combined with the scaffolding and educational design of the curriculum materials. This facilitates their non-trivial authentic use of images, whether for science-grade photometry or for simple colour imaging.

- Increase students' understanding and appreciation for the universe around them, what it looks like, what its history is and where they are in it as far as we can currently ascertain;
In terms of simple conceptual content knowledge, evidence shows that we are making an impact. We are currently in the process of collecting and examining the outcomes from the more deeper assessments of whether students have a broader understanding of the nature and history of the universe.

- Increase students' appreciation for the true methodology and approach of science in contrast to the general, currently poor, students' perceptions of school science;

As yet, we are uncertain about this. We have developed a new instrument to probe student attitudes and perceptions of science and astronomy (Bartlett et al., 2018) but it is only early days for the instrument. We hope to be making a positive impact, but, as we found in our previous study (Fitzgerald et al., 2016) we suspect that we will have a lot to learn as time goes on about this aspect of the project. It is well known that shifting student attitudes is a very difficult process in comparison to simple content knowledge gains.

- Increase the probability of students choosing science, other than as a potential personal interest, as a topic for higher level study or 
as a potential future career path or, at the very least, help them discover they are actually interested in science; and,

We have undertaken no robust assessment of this. We have anecdotes that we can share about certain teachers and classes and individual students, but not enough anecdotes that we can say that it is entirely a common theme. We have also incorporated career intentions into the recently created student attitude survey to probe this also.

- Enable students, or a smaller subset that so desire, to take their research to a natural scientific conclusion. Sometimes even to result in a scientific publication.

This is something the project has had success with, with a number of science publications achieved and a number of projects sufficiently completed and concluded.

Our Solar Siblings is now looking to expand to a greater scale. There are three particular aspects in this future growth: increasing efficiency, ruggedizing content, and spreading the word. In order to increase viability of the program, we need to focus on minimising the time spent by project personnel dealing with the mundane aspects of data by the use of the OSS Pipeline (as described in Fitzgerald 2018, these proceedings). To increase robustness and consistency within the program, we need to continue development of appropriate bulletproof, road-tested working curriculum material which fits the new Australian curriculum. And finally, no project is successful unless someone uses it! We intend to carry out a major promotional drive to recruit teachers, schools and districts towards the end of 2017 in preparation for the Australian school year starting in early 2018.

\section{References}

Australian Curriculum Assessment and Reporting Authority (2017). The Australian Curriculum: Science. Retrieved from http://www.australiancurriculum.edu.au/.
Bartlett, S., Fitzgerald, M. T., McKinnon, D. H., Danaia, L., and Lazendic-Galloway, J. (2018). Astronomy And Science Student Attitudes (ASSA): A Short Review And Validation Of A New Instrument. Journal of Astronomy \& Earth Sciences Education-June, 5(1).

Brown, T., Baliber, N., Bianco, F., Bowman, M., Burleson, B., Conway, P., Crellin, M., Depagne, É., De Vera, J., Dilday, B., et al. (2013). Las Cumbres Observatory global telescope network. Publications of the Astronomical Society of the Pacific, 125(931):1031.

Cáceres, C. and Catelan, M. (2008). The periodluminosity relation of RR Lyrae stars in the SDSS photometric system. The Astrophysical Journal Supplement Series, 179(1):242.

Catelan, M., Torrealba, G. I., Cáceres, C., Smith, H. A., De Lee, N., and Fitzgerald, M. (2012). Physical parameters for RR Lyrae stars in the sdss filter system. Proceedings of the International Astronomical Union, 8(S289):126-129.

Collins, K. A., Kielkopf, J. F., Stassun, K. G., and Hessman, F. V. (2017). AstroImageJ: image processing and photometric extraction for ultraprecise astronomical light curves. The Astronomical Journal, 153(2):77.

Cutts, K. R. (2018). Astronomy Student Research in the International Baccalaureate. In Fitzgerald, M. T., James, C. R., Buxner, S., and White, S., editors, Robotic Telescopes, Student Research, and Education Proceedings, volume 1(1), pages 299-315.

Danaia, L., Fitzgerald, M., and McKinnon, D. (2013). Students' perceptions of high school science: what has changed over the last decade? $R e$ search in Science Education, 43(4):1501-1515.

Danaia, L., McKinnon, D., Parker, Q., Fitzgerald, M., and Stenning, P. (2012). Space to grow: LCOGT.net and improving science engagement in schools. Astronomy Education Review, 11(1). 
Danaia, L., McKinnon, D. H., and Fitzgerald, M. (2017). Ideal pictures and actual perspectives of junior secondary school science: comparisons drawn from Australian students in an astronomy education programme. Research in Science \& Technological Education, 35(4):445-460.

Dias, W., Alessi, B., Moitinho, A., and Lépine, J. (2002). New catalogue of optically visible open clusters and candidates. Astronomy \& Astrophysics, 389(3):871-873.

Fitzgerald, M. (2018). The Our Solar Siblings Pipeline: Tackling the data issues of the scaling problem for robotic telescope based astronomy education projects. In Fitzgerald, M. T., James, C. R., Buxner, S., and White, S., editors, Robotic Telescopes, Student Research, and Education Proceedings, volume 1(1), pages 343354.

Fitzgerald, M., Danaia, L., and McKinnon, D. H. (2017). Barriers inhibiting inquiry-based science teaching and potential solutions: perceptions of positively inclined early adopters. Research in Science Education, pages 1-24.

Fitzgerald, M., McKinnon, D. H., and Danaia, L. (2015a). Inquiry-based educational design for large-scale high school astronomy projects using real telescopes. Journal of Science Education and Technology, 24(6):747-760.

Fitzgerald, M., McKinnon, D. H., Danaia, L., and Deehan, J. (2016). A large-scale inquiry-based astronomy intervention project: impact on students' content knowledge performance and views of their high school science classroom. Research in Science Education, 46(6):901-916.

Fitzgerald, M. T., Criss, J., Lukaszewicz, T., Frew, D. J., Catelan, M., Woodward, S., Danaia, L., and McKinnon, D. H. (2012). RR Lyrae stars in the globular cluster NGC 6101. Publications of the Astronomical Society of Australia, 29(1):72-77.

Fitzgerald, M. T., Hollow, R., Rebull, L. M., Danaia, L., and McKinnon, D. H. (2014). A review of high school level astronomy student research projects over the last two decades. Publications of the Astronomical Society of Australia, 31.

Fitzgerald, M. T., Inwood, L., McKinnon, D. H., Dias, W., Sacchi, M., Scott, B., Zolinski, M., Danaia, L., and Edwards, R. (2015b). Photometric and proper motion study of the neglected open cluster NGC 2215. The Astronomical Journal, 149(6):190.

Girardi, L., Bertelli, G., Bressan, A., Chiosi, C., Groenewegen, M., Marigo, P., Salasnich, B., and Weiss, A. (2002). Theoretical isochrones in several photometric systems-I. Johnson-CousinsGlass, HST/WFPC2, HST/NICMOS, Washington, and ESO Imaging Survey filter sets. Astronomy \& Astrophysics, 391(1):195-212.

Girardi, L., Grebel, E. K., Odenkirchen, M., and Chiosi, C. (2004). Theoretical isochrones in several photometric systems-II. The Sloan Digital Sky Survey ugriz system. Astronomy \& Astrophysics, 422(1):205-215.

Gomez, E. L. and Fitzgerald, M. T. (2017). Robotic telescopes in education. Astronomical Review, 13(1):28-68.

Karataş, Y. and Schuster, W. (2006). Metallicity and absolute magnitude calibrations for UBV photometry. Monthly Notices of the Royal Astronomical Society, 371(4):1793-1812.

Laher, R. R., Gorjian, V., Rebull, L. M., Masci, F. J., Fowler, J. W., Helou, G., Kulkarni, S. R., and Law, N. M. (2012). Aperture photometry tool. Publications of the Astronomical Society of the Pacific, 124(917):737.

Lazendic-Galloway, J., Fitzgerald, M., and McKinnon, D. H. (2017). Implementing a Studio-based Flipped Classroom in a First Year Astronomy Course. International Journal of Innovation in Science and Mathematics Education (formerly CAL-laborate International), 24(5):35-47. 
Mighell, K. (1999). CCD aperture photometry. In Precision CCD Photometry, volume 189, page 50 .

Monteiro, H., Dias, W., and Caetano, T. (2010). Fitting isochrones to open cluster photometric data-A new global optimization tool. Astronomy \& Astrophysics, 516:A2.

Paunzen, E. and Netopil, M. (2006). On the current status of open-cluster parameters. Monthly Notices of the Royal Astronomical Society, 371(4):1641-1647.

Rebull, L. (2018). Authentic Research in the Classroom for Teachers and Students. In Fitzgerald, M. T., James, C. R., Buxner, S., and White, S., editors, Robotic Telescopes, Student Research, and Education Proceedings, volume 1(1), pages 21-31.

Schleigh, S. P., Slater, S. J., Slater, T. F., and Stork, D. J. (2015). The new curriculum standards for astronomy in the United States. Latin American Journal of Astronomy Education, 20:131-151.

Slater, S. J., Slater, T. F., and Shaner, A. (2008). Impact of backwards faded scaffolding in an astronomy course for pre-service elementary teachers based on inquiry. Journal of Geoscience Education, 56(5):408-416.

Slater, T. F., Burrows, A. C., French, D. A., Sanchez, R. A., and Tatge, C. B. (2014). A proposed astronomy learning progression for remote telescope observation. Journal of College Teaching \& Learning (Online), 11(4):197.

Souza, S. (2013). Two new cool variable stars in the field of NGC 659. Journal of the American Association of Variable Star Observers (JAAVSO), 41:92.

Stetson, P. B. (1987). DAOPHOT: A computer program for crowded-field stellar photometry. Publications of the Astronomical Society of the Pacific, 99(613):191.
Templeton, M., Henden, A., Goff, W., Smith, S., Sabo, R., Walker, G., Buchheim, R., Belcheva, G., Crawford, T., Cook, M., et al. (2011). Modern observations of Hubble's first-discovered Cepheid in M31. Publications of the Astronomical Society of the Pacific, 123(910):1374.

Thomson, S., De Bortoli, L., and Underwood, C. (2016a). PISA 2015: A first look at Australia's results.

Thomson, S., Wernert, N., O'Grady, E., and Rodrigues, S. (2016b). TIMSS 2015: A first look at Australia's results.

Townsend, A., McKinnon, D. H., Fitzgerald, M. T., Morris, J., and Lummis, G. (2017). Educative curricula and PCK development driven by STEM professional learning in rural and remote schools: a longitudinal Type IV Case Study. International Journal of Innovation in Science and Mathematics Education, 24(4). 